\title{
COMPACT DIFFERENCES OF COMPOSITION OPERATORS
}

\author{
ELKE WOLF
}

(Received 7 June 2007)

\begin{abstract}
Let $\phi$ and $\psi$ be analytic self-maps of the open unit disk. Each of them induces a composition operator, $C_{\phi}$ and $C_{\psi}$ respectively, acting between weighted Bergman spaces of infinite order. We show that the difference $C_{\phi}-C_{\psi}$ is compact if and only if both operators are compact or both operators are not compact and the pseudohyperbolic distance of the functions $\phi$ and $\psi$ tends to zero if $|\phi(z)| \rightarrow 1$ or $|\psi(z)| \rightarrow 1$.
\end{abstract}

2000 Mathematics subject classification: 47B33, 47B38.

Keywords and phrases: differences of weighted composition operators, compact, weighted Bergman spaces of infinite order.

\section{Introduction}

Let $\phi, \psi: D \rightarrow D$ analytic maps. Each such map induces through composition a linear composition operator $C_{\phi}(f)=f \circ \phi$ and $C_{\psi}(f)=f \circ \psi$. Let $v$ and $w$ be strictly positive continuous and bounded functions (weights) on the unit disk $D$ of the complex plane. We are interested in differences of composition operators defined on weighted Bergman spaces of infinite order,

$$
H_{v}^{\infty}:=\left\{f \in H(D) ;\|f\|_{v}=\sup _{z \in D} v(z)|f(z)|<\infty\right\}
$$

where $H(D)$ denotes the space of all analytic functions on $D$.

In [7] MacCluer et al. showed that the difference of two composition operators acting on the space $H^{\infty}(D)$ of bounded analytic functions on $D$ is compact if and only if both operators are compact or if both operators are not compact and the pseudohyperbolic distance of the analytic self-maps $\phi$ and $\psi$ of $D$ tends to zero if $|\phi(z)| \rightarrow 1$ or $|\psi(z)| \rightarrow 1$. The aim of this article is to show that under some assumptions on the weights this remains true in the setting of weighted Bergman spaces of infinite order. In [3] we already characterized the essential norm and

(c) 2008 Australian Mathematical Society 0004-9727/08 \$A2.00+0.00 
therefore also the compactness of the difference of composition operators in terms of the weights. Here we want to show how compactness of the difference is related to the compactness of the single composition operators.

\section{Notation and auxiliary results}

For notation on composition operators we refer the reader to the monographs [4] and [8]. For differences of composition operators we need the pseudohyperbolic metric $\rho(z, w):=\left|\varphi_{z}(w)\right|$ for $z, w \in D$, where $\varphi_{z}(w)=(z-w) /(1-\bar{z} w)$ is the Möbius transformation of $D$.

Let $B_{v}^{\infty}$ be the closed unit ball of the space $H_{v}^{\infty}$. Very often the so-called associated weights are needed to formulate results on weighted Bergman spaces of infinite order. For a weight $v$ the associated weight $\tilde{v}$ is given by

$$
\tilde{v}(z)=1 /\left(\sup \left\{|f(z)|, f \in B_{v}^{\infty}\right\}\right)=1 /\left\|\delta_{z}\right\|_{H_{v}^{\infty}},
$$

where $\delta_{z}$ denotes the point evaluation of $z$. By [1] we know that the associated weight $\tilde{v}$ has the following properties:

(i) $\tilde{v}$ is continuous;

(ii) $\tilde{v}>v>0$;

(iii) for every $z \in D$ we can find $f_{z} \in B_{v}^{\infty}$ such that $\left|f_{z}(z)\right|=1 /(\tilde{v}(z))$.

We are especially interested in the radial weights which satisfy the following condition (which is due to Lusky, see [6]):

$$
\inf _{k} \frac{v\left(1-2^{-k-1}\right)}{v\left(1-2^{-k}\right)}>0 .
$$

Lusky showed that each of the following weights satisfies the condition (L1):

$$
\begin{gathered}
v_{p}(z)=(1-|z|)^{p}, \quad 0<p<\infty, \\
w_{1}(z)=(1-\log (1-|z|))^{-\beta}, \quad \beta>0, \\
w_{2}(z)=(1-|z|)^{p}(1-\log (1-|z|))^{-\beta}, \quad 0<p<\infty, \beta>0 .
\end{gathered}
$$

Radial weights with $\lim _{|z| \rightarrow 1} v(z)=0$ are called typical weights.

For a better understanding we want to collect some auxiliary results that we need to prove our main result.

THEOREM 1 (Bonet et al. [2]). Let $\phi$ be an analytic self-map of D.

(a) Let $v$ and $w$ be radial weights. Then the following assertions are equivalent.

(i) The operator $C_{\phi}: H_{v}^{\infty} \rightarrow H_{w}^{\infty}$ is bounded.

(ii) $\sup _{z \in D}(w(z)) /(\tilde{v}(\phi(z)))=M<\infty$.

(b) Let $v$ and $w$ be typical weights. Then the following statements are equivalent.

(i) The operator $C_{\phi}: H_{v}^{\infty} \rightarrow H_{w}^{\infty}$ is compact.

(ii) $\lim _{|z| \rightarrow 1}(w(z)) /(\tilde{v}(\phi(z)))=0$. 
LEMMA 2 (Bonet et al. [3]). Let $v$ be a radial weight satisfying the Lusky condition (L1) and let $f \in H_{v}^{\infty}$. Then there exists a constant $C_{v}$ (depending on the weight $v$ ) such that

$$
|f(z)-f(p)| \leq C_{v}\|f\|_{v} \max \left\{\frac{1}{v(z)}, \frac{1}{v(p)}\right\} \rho(z, p),
$$

for all $z, p \in D$.

LEMMA 3 (Lindström and Wolf [5]). Let $v$ be a radial weight on D satisfying the Lusky condition (L1) such that $v$ is continuously differentiable with respect to $|z|$. Then $v(p) / v(z) \rightarrow 1$ when $\rho(p, z) \rightarrow 1$.

Theorem 4 (Cowen and MacCluer [4, Proposition 3.11]). Let $\phi$ and $\psi$ be analytic self-maps of the disk. If $C_{\phi}-C_{\psi}: H_{v}^{\infty} \rightarrow H_{w}^{\infty}$ is bounded, then $C_{\phi}-C_{\psi}$ : $H_{v}^{\infty} \rightarrow H_{w}^{\infty}$ is compact if and only if whenever $\left(h_{n}\right)_{n \in \mathbb{N}}$ is a bounded sequence in $H_{v}^{\infty}$ with $h_{n} \rightarrow 0$ uniformly on compact subsets of $D$, then $\left\|\left(C_{\phi}-C_{\psi}\right) h_{n}\right\|_{w} \rightarrow 0$.

\section{Main result}

THEOREM 5. Let $v$ and $w$ be typical weights such that $v$ is continuously differentiable with respect to $|z|$ and satisfies $(L 1)$. We assume that $C_{\phi}, C_{\psi}: H_{v}^{\infty} \rightarrow$ $H_{w}^{\infty}$ both are bounded. Then $C_{\phi}-C_{\psi}: H_{v}^{\infty} \rightarrow H_{w}^{\infty}$ is compact if and only if one of (a) or (b) holds:

(a) $C_{\phi}$ and $C_{\psi}$ are compact;

(b) $C_{\phi}$ and $C_{\psi}$ both are not compact and

$$
\lim _{|\phi(z)| \rightarrow 1} \rho(\phi(z), \psi(z))=\lim _{|\psi(z)| \rightarrow 1} \rho(\phi(z), \psi(z))=0 .
$$

Proof. Suppose (a) is not true. Without loss of generality, we may assume that $C_{\phi}$ is not compact.

Moreover, first we assume that $\lim _{|\phi(z)| \rightarrow 1} \rho(\phi(z), \psi(z)) \neq 0$. Then there is a sequence $\left(z_{n}\right)_{n \in \mathbb{N}} \subset D$ such that $\left|\phi\left(z_{n}\right)\right| \rightarrow 1$ (and hence $\left|z_{n}\right| \rightarrow 1$ ) and

$$
\rho\left(\phi\left(z_{n}\right), \psi\left(z_{n}\right)\right) \geq \alpha>0 \quad \text { for every } n \in \mathbb{N} \text {. }
$$

Furthermore, since $C_{\phi}$ is not compact and $v$ and $w$ both are radial, by Theorem 1 it follows that $w\left(z_{n}\right) / \tilde{v}\left(\phi\left(z_{n}\right)\right) \geq \beta>0$ for every $n \in \mathbb{N}$. Next we choose an increasing sequence $\left(\alpha_{n}\right)_{n \in \mathbb{N}}$ of natural numbers going to infinity such that $\left|\phi\left(z_{n}\right)\right|^{\alpha_{n}} \geq \gamma>0$ for every $n \in \mathbb{N}$. For every $n \in \mathbb{N}$ we can find $f_{n} \in B_{v}^{\infty}$ such that $\left|f_{n}\left(\phi\left(z_{n}\right)\right)\right|=$ $1 / \tilde{v}\left(\phi\left(z_{n}\right)\right)$. Now set

$$
h_{n}(z):=z^{\alpha_{n}} \varphi_{\psi\left(z_{n}\right)}(z) f_{n}(z)
$$


Hence $\left(h_{n}\right)_{n} \subset B_{v}^{\infty}$ and $\left(h_{n}\right)_{n}$ tends to zero on the compact sets. Since $C_{\phi}-C_{\psi}$ : $H_{v}^{\infty} \rightarrow H_{w}^{\infty}$ is compact, by Theorem $4\left(\left\|\left(C_{\phi}-C_{\psi}\right) h_{n}\right\|_{w}\right)_{n \in \mathbb{N}}$ must tend to zero. But

$$
\left\|\left(C_{\phi}-C_{\psi}\right) h_{n}\right\|_{w} \geq\left|\phi\left(z_{n}\right)\right|^{\alpha_{n}} \frac{w\left(z_{n}\right)}{\tilde{v}\left(\phi\left(z_{n}\right)\right)} \rho\left(\phi\left(z_{n}\right), \psi\left(z_{n}\right)\right) \geq \alpha \beta \gamma>0 .
$$

This is a contradiction.

Next, we assume that $\lim _{|\psi(z)| \rightarrow 1} \rho(\phi(z), \psi(z)) \neq 0$. Then there is a sequence $\left(z_{n}\right)_{n \in \mathbb{N}} \subset D$ such that $\left|\psi\left(z_{n}\right)\right| \rightarrow 1$ (and hence $\left|z_{n}\right| \rightarrow 1$ and thus $\left|\phi\left(z_{n}\right)\right| \rightarrow 1$ ) and

$$
\rho\left(\phi\left(z_{n}\right), \psi\left(z_{n}\right)\right) \geq \alpha>0 \quad \text { for every } n \in \mathbb{N} .
$$

Since $w$ and $v$ are radial and $C_{\phi}$ is not compact, Theorem 1 yields

$$
w\left(z_{n}\right) / v\left(\phi\left(z_{n}\right)\right) \geq \beta>0
$$

for every $n \in \mathbb{N}$. From now on the proof is analogous to the proof of the previous case.

It remains to show that $C_{\psi}$ is not compact. By Lemma 3 we know that

$$
\left|\frac{\tilde{v}(\phi(z))}{\tilde{v}(\psi(z))}-1\right| \rightarrow 0 \quad \text { if } \rho(\phi(z), \psi(z)) \rightarrow 0 .
$$

Hence $C_{\psi}$ is not compact, since $C_{\phi}$ is not compact.

For the converse, by Theorem 4 we have to show $\lim _{n \rightarrow \infty}\left\|\left(C_{\phi}-C_{\psi}\right) h_{n}\right\|_{w}=0$ for $\left(h_{n}\right)_{n \in \mathbb{N}}$ bounded and $h_{n} \rightarrow 0$ uniformly on compact subsets of $D$. It is enough to consider $\left(h_{n}\right)_{n \in \mathbb{N}} \subset B_{v}^{\infty}$. By Lemma 2 we can find $C>0$ such that

$$
\left|h_{n}(\phi(z))-h_{n}(\psi(z))\right| \leq C \max \left\{\frac{1}{\tilde{v}(\phi(z))}, \frac{1}{\tilde{v}(\psi(z))}\right\} \rho(\phi(z), \psi(z)) .
$$

By Theorem 1 and the assumption that $C_{\phi}$ and $C_{\psi}$ are bounded, we can choose $M>0$ such that $\sup _{z \in D} w(z) \max \{1 / \tilde{v}(\phi(z)), 1 / \tilde{v}(\psi(z))\} \leq M$. Given $\varepsilon>0$ we may find $r<1$ such that

$$
\begin{aligned}
& |\phi(z)| \geq r \Longrightarrow \rho(\phi(z), \psi(z))<\frac{\varepsilon}{C M} \text { and } \\
& |\psi(z)| \geq r \Longrightarrow \rho(\phi(z), \psi(z))<\frac{\varepsilon}{C M} .
\end{aligned}
$$

Thus, we obtain

$$
w(z)\left|h_{n}(\phi(z))-h_{n}(\psi(z))\right|<\varepsilon \quad \text { if }|\phi(z)| \geq r \text { or }|\psi(z)| \geq r,
$$

and, by almost uniform convergence of $\left(h_{n}\right)_{n \in \mathbb{N}}$ to zero and boundedness of $w$, we can find $n_{0} \in \mathbb{N}$ such that

$$
w(z)\left|h_{n}(\phi(z))-h_{n}(\psi(z))\right|<\varepsilon \quad \text { for } n \geq n_{0},|\psi(z)| \leq r,|\phi(z)| \leq r .
$$

Hence the claim follows. 
For examples on compact and non-compact differences of composition operators acting between weighted Bergman spaces of infinite order we refer the reader to [3].

\section{References}

[1] K. D. Bierstedt, J. Bonet and J. Taskinen, 'Associated weights and spaces of holomorphic functions', Studia Math. 127 (1998), 137-168.

[2] J. Bonet, P. Domański, M. Lindström and J. Taskinen, 'Composition operators between weighted Banach spaces of analytic functions', J. Austral. Math. Soc. Ser. A 64 (1998), 101-118.

[3] J. Bonet, M. Lindström and E. Wolf, 'Differences of composition operators between weighted Banach spaces of holomorphic functions'. J. Austral. Math. Soc. to appear.

[4] C. Cowen and B. MacCluer, Composition operators on spaces of analytic functions (CRC Press, Boca Raton, FL, 1995).

[5] M. Lindström and E. Wolf, 'Essential norm of the difference of weighted composition operators', Monatshefte Math. 153 (2008), 133-143.

[6] W. Lusky, 'On weighted spaces of harmonic and holomorphic functions', J. London Math. Soc. 51 (1995), 309-320.

[7] B. MacCluer, S. Ohno and R. Zhao, 'Topological structure of the space of composition operators on $H^{\infty}$, Integral Equations Operator Theory 40(4) (2001), 481-494.

[8] J. H. Shapiro, Composition operators and classical function theory (Springer, Berlin, 1993).

\section{Mathematical Institute \\ University of Paderborn \\ D-33095 Paderborn}

Germany

e-mail: lichte@ math.uni-paderborn.de 